Gut and Liver, Vol. 12, No. 4, July 2018, pp. 373-374

\title{
Does Estrogen Contribute to the Esophageal Barrier Function in Women?
}

\author{
Katsunori lijima \\ Department of Gastroenterology, Akita University Graduate School of Medicine, Akita, Japan
}

\begin{abstract}
See "Comparison of Tight Junction Protein-Related Gene mRNA Expression Levels between Male and Female Gastroesophageal Reflux Disease Patients" by Jin Joo Kim, et al. on page 411-419, Vol. 12. No. 4, 2018
\end{abstract}

Esophageal adenocarcinoma (EAC) develops as a series on the gastroesophageal reflux disease (GERD) spectrum, progressing from reflux esophagitis to Barrett's esophagus and finally EAC. EAC has received intense focus for decades, especially in Western countries, where the incidence of EAC been increasing since the 1970s. However, in Asian countries, there has been no definite increasing trend with regard to EAC studies, with the proportion of EAC accounting for only a small percentage of total esophageal cancer cases. ${ }^{1}$ However, a very recent study from Japan indicated that the incidence of EAC has started to increase in Japan, ${ }^{2}$ due at least in part to a decreasing trend in the Helicobacter pylori infection rate in the general Japanese population, which is lagging 50 years behind Western countries.

One prominent characteristic of EAC is its overwhelming male predominance, as described in a recent review. ${ }^{3}$ Furthermore, such male predominance is already initiated at the initial stage of the GERD spectrum, e.g., reflux esophagitis, although the tendency is amplified as the disease spectrum progresses. ${ }^{3}$ Hence, understanding the mechanism underlying the male predominance of reflux esophagitis may be important not only for the treatment of reflux esophagitis, but also for the prevention of the most advanced stage of GERD, EAC. A recent animal model study showed that the female hormone estrogen plays an important role in protecting the esophageal tissue from refluxed liminal noxious agents. ${ }^{4}$ In addition, another study indicated that estrogen enhances the epithelial barrier function of the esophagus by potentiating a tight-junction protein, occludin, consequently preventing luminal noxious agents from entering the esophageal epithelium. ${ }^{5}$ Consistent with these animal model studies, many epidemiological studies support the potentially pivotal role of estrogen in the esophageal barrier function. ${ }^{3}$ For example, the prevalence of reflux esophagitis is suppressed in premenopausal women compared with that in men; however, the prevalence steadily increases in postmenopausal women to a level similar to that in men. Furthermore, the prevalence of reflux esophagitis is relatively stable in all age groups in men. ${ }^{3}$ These epidemiological studies also indicate that esophageal mucosal injury is suppressed in premenopausal women by the protective action of estrogen. However, direct evidence that estrogen enhances the esophageal barrier function in human remains elusive.

In the present issue of Gut and Liver, Kim et al. ${ }^{6}$ investigated the expression of tight-junction-related protein mRNA in male and female GERD patients and found several key differences in the mRNA expression according to the severity of the disease (e.g., erosive reflux esophagitis vs nonerosive reflux esophagitis vs controls). However, they failed to find any significant difference in the mRNA levels between genders. ${ }^{6}$ Unfortunately, the patients enrolled in their study appear to have been unsuitable for an investigation into the effects of estrogen on the esophageal barrier function, as the authors acknowledged in the article, as only three premenopausal women with controls and no premenopausal women with GERD were included in that study. ${ }^{6}$ Thus, studies enrolling premenopausal patients with GERD will be required to investigate the potential effects of estrogen on the esophageal barrier function.

In addition, several methodological issues remain to be resolved. As the authors described in the article, not only the amount of the protein mRNA but also the localization of the proteins may be important for the maintenance of the esophageal barrier function. Furthermore, given that the mRNA level can be up- or down-regulated, depending on the degree of concomitant inflammation of the esophageal epithelium, the interpretation of such results may be complicated. In this

Correspondence to: Katsunori Iijima

Department of Gastroenterology, Akita University Graduate School of Medicine, 1-1-1 Hondo, Akita 010-8543, Japan

Tel: +81-18-884-6573, Fax: +81-18-801-7501, E-mail: kiijima@med.akita-u.ac.jp

pISSN 1976-2283 eISSN 2005-1212 https://doi.org/10.5009/gnl18199

@ This is an Open Access article distributed under the terms of the Creative Commons Attribution Non-Commercial License (http://creativecommons.org/licenses/by-nc/4.0) which permits unrestricted non-commercial use, distribution, and reproduction in any medium, provided the original work is properly cited. 
sense, the dilatation of the inter-cellular space might be a more straightforward biomarker of the esophageal barrier function. ${ }^{5}$ Finally, the results from chronically diseased patients may be confounded by concomitant inflammation or the duration of the disease. Therefore, the instillation of $\mathrm{HCl}$ to the esophagus in healthy men and women (especially premenopausal women) with a comparison of the reactive esophageal barrier function between genders may be a more direct method of evaluating the role of estrogen on the esophageal barrier function under physiological conditions, although the pathological implications of such a study remain to clarified.

Aside from enhancing the esophageal barrier function, estrogen exerts a variety of anti-inflammatory effects on the gastrointestinal tract, as described in a recent review. ${ }^{7}$ A very recent study showed that estrogen enhances the host defense mechanism against oxidative stress induced by esophagitis by enhancing the expression of nuclear factor erythroid 2-related factor 2 (Nrf2), a potent transcriptional factor in the host defense mechanism. ${ }^{8}$

The potential protective effects of estrogen on the esophageal epithelium against noxious luminal agents will have therapeutic implications, as these effects imply that the hormone can be used for the chemoprevention of EAC by suppressing the initial step of the GERD spectrum, reflux esophagitis. Indeed, a recent study successfully demonstrated that menopausal hormone therapy reduces the risk of EAC. ${ }^{9}$ In contrast, hormonereplacement therapy may exacerbate GERD symptoms, probably by enhancing the sensitivity of the esophageal epithelium to the stimuli. ${ }^{10}$ Thus, further studies to investigate the precise mechanism underlying the protective effects of estrogen on the esophageal epithelium are required to strike a balance between the beneficial and harmful effects of estrogen in managing GERD patients.

\section{CONFLICTS OF INTEREST}

No potential conflict of interest relevant to this article was reported.

\section{REFERENCES}

1. Rubenstein JH, Shaheen NJ. Epidemiology, diagnosis, and management of esophageal adenocarcinoma. Gastroenterology 2015; 149:302-317.e1.

2. Koizumi S, Motoyama S, Iijima K. Is the incidence of esophageal adenocarcinoma increasing in Japan? Trends from the data of a hospital-based registration system in Akita Prefecture, Japan. J Gastroenterol. Epub 2017 Nov 14. https://doi.org/10.1007/s00535017-1412-4.

3. Asanuma K, Iijima K, Shimosegawa T. Gender difference in gastroesophageal reflux diseases. World J Gastroenterol 2016;22:18001810.

4. Masaka T, Iijima K, Endo H, et al. Gender differences in oesophageal mucosal injury in a reflux oesophagitis model of rats. Gut 2013;62:6-14.

5. Honda J, lijima K, Asanuma K, et al. Estrogen enhances esophageal barrier function by potentiating occludin expression. Dig Dis Sci 2016;61:1028-1038.

6. Kim JJ, Kim N, Park JH, et al. Comparison of tight junction protein-related gene mRNA expression levels between male and female gastroesophageal reflux disease patients. Gut Liver 2018;12:411-419.

7. Nie X, Xie R, Tuo B. Effects of estrogen on the gastrointestinal tract. Dig Dis Sci 2018;63:583-596.

8. Torihata Y, Asanuma K, Iijima K, et al. Estrogen-dependent Nrf2 expression protects against reflux-induced esophagitis. Dig Dis Sci 2018;63:345-355.

9. Brusselaers N, Maret-Ouda J, Konings P, El-Serag HB, Lagergren J. Menopausal hormone therapy and the risk of esophageal and gastric cancer. Int J Cancer 2017;140:1693-1699.

10. Jacobson BC, Moy B, Colditz GA, Fuchs CS. Postmenopausal hormone use and symptoms of gastroesophageal reflux. Arch Intern Med 2008;168:1798-1804. 